\title{
Electrical transport in AZO nanorods
}

A. Yildiz ${ }^{1 *}$, H. Cansizoglu ${ }^{2}$ and T. Karabacak ${ }^{2}$

${ }^{1}$ Department of Energy Systems Engineering, Faculty of Engineering and Natural Sciences, Yıldırım Beyazıt University, Ankara, Turkey

${ }^{2}$ Department of Physics and Astronomy, University of Arkansas at Little Rock, Little Rock, Arkansas, 72204, USA

* Corresponding author: tel.: +90 31232415 55; fax: +90 31232415 05; e-mail address: yildizab@gmail.com

\begin{abstract}
Al-doped $\mathrm{ZnO}(\mathrm{AZO})$ nanorods (NRs) with different lengths were deposited by utilizing glancing angle deposition (GLAD) technique in a DC sputter system at room temperature. The structural and optical characteristics of the NRs were investigated by the X-ray diffraction (XRD), scanning electron microscopy (SEM), and UV-vis-NIR spectroscopy measurements. A band gap of about $3.5 \mathrm{eV}$ was observed for the NRs. A novel capping process utilizing varying deposition angles was used to introduce a blanket metal top contact for the electrical characterization of NRs. Current-voltage (I-V) measurements were used to properly evaluate the approximate resistivity of a single NR. The electrical conduction was found to be governed by the thermally activated transport mechanism. Activation energy was determined as $0.14 \mathrm{eV}$ from temperature dependent resistivity data.
\end{abstract}

\section{Keywords}

AZO Nanorods; Glancing Angle Deposition (GLAD); Electrical Properties; Activation Energy 


\section{Introduction}

The unique optical, electrical properties and structural diversity of one-dimensional nanostructures have triggered considerable research attention in different fields of nanoscale electronic and optoelectronic devices [1,2]. Having a band gap of $3.37 \mathrm{eV}$ and a large exciton binding energy of $60 \mathrm{meV}$ makes $\mathrm{ZnO}$ a suitable candidate for these types of devices. $\mathrm{ZnO}$ nanowires and nanorods have a number of properties desirable for various device applications $[3,4]$. A literature survey reveals that most of the work focuses on the synthesis of $\mathrm{ZnO}$ nanostructures with various shapes [5,6]. Even less is known about the electrical properties of $\mathrm{ZnO}$ nanorods (NRs). Estimation of electrical resistivity of an individual nanorod can provide valuable information about electrical properties of the ZnO NRs.

In this work, we employed a simple method to determine approximate electrical resistivity of a single $\mathrm{Al}$-doped $\mathrm{ZnO}$ nanorod deposited by glancing angle deposition (GLAD) method at room temperature. We utilized a new capping process to introduce a blanket layer of metal contact on nanorod arrays.

\section{Experimental}

Vertical arrays of AZO NRs were grown on $\mathrm{Si}$, silver coated $\mathrm{Si}$, and glass substrates by GLAD in a DC sputtering system. The $\mathrm{ZnO}-\mathrm{Al}$ (2 wt.\%) target (Kurt Lesker) was used as the source material. All of the samples were deposited at room temperature under $6 \times 10^{-6} \mathrm{mbar}$ base pressure with a DC power of $110 \mathrm{~W}$. The working gas (Ar) pressure was $3.5 \times 10^{-3} \mathrm{mbar}$. The NRs were deposited under identical experimental conditions except the deposition time. In order to get different NR lengths of about 300, 385, 470 and $545 \mathrm{~nm}$, the deposition times were set to $84,105,126$, and $146 \mathrm{~min}$ and $120 \mathrm{~min}$, respectively. The incidence angle of the flux and the substrate rotation speed for GLAD were set at about $\sim 90^{\circ}$ and 0.2 rotation per minute (rpm), respectively. 
$\theta-2 \theta$ scan X-ray diffraction (XRD) profiles of AZO NRs on $\mathrm{Si}$ substrates were obtained in a Rigaku MiniFlex 600 unit using $1.54 \mathrm{~A}^{\mathrm{o}}$ wavelength X-ray source. The morphological characterization of the samples were conducted by scanning electron microscopy (SEM, JEOL 7000F). Identical AZO NRs deposited on glass substrates were used for optical characterization. The total optical reflection and transmission profiles of the samples were obtained by UV-vis-NIR spectroscopy measurements by utilizing an integrating sphere (Shimadzu UV-3600). The light source was incident at a near-normal angle of $8^{\circ}$ on the samples for reflectance measurements. Temperature dependent I-V measurements were taken by two probes connected to Ketihley 2400 Sourcemeter. AZO nanorods grown on Agfilm/Si substrates were used for the $\mathrm{I}-\mathrm{V}$ measurements. However, to eliminate the short circuitry between top and bottom contacts, a unique capping process was utilized to deposit a blanket metal layer on the tips of the nanorods. The details of the capping process can be found in Ref [7].

\section{Results and Discussion}

Figure 1 shows the XRD patterns of the AZO NRs. Only a main peak located at about $34.4^{\circ}$ was observed for all the samples. It is assigned to the (002) plane, indicating that the samples have a preferential [002] orientation along the normal direction of the substrate surface. The intensity of (002) diffraction peak increases as the length of the NRs increases (see inset of Fig. 1), which is consistent with the previously reported results [8]. Fig. 2 depicts the top-view and cross-sectional SEM images of the NRs which have diameters of about $70,65,85$, and $90 \mathrm{~nm}$ for NR lengths of 300, 385, 470, and $545 \mathrm{~nm}$, respectively. Fig. 1 reveals that most of the samples are vertically well-aligned and densely packed.

The optical band gaps of the NRs were determined on the basis of UV-Vis-NIR transmission measurements. The optical band gap of films can be estimated using the relation 
between absorption coefficient and energy band gap $(\alpha h v)^{2}=B\left(h v-E_{g}\right)$, where $h v, E_{g}$, and $B$ are the incident photon energy, the optical band gap energy, and a constant, respectively [9]. The linear portion is extrapolated to $\alpha=0$, on energy axis, which gives the band gap energy (Fig. 3). It is about $3.5 \mathrm{eV}$ and has small variations with the length of the NRs.

Fig. 4 shows the $I-V$ characteristics as a function of temperature. The inset of Fig. 4 shows the corresponding schematic current measurement set-up. The NRs exhibit linear behaviors indicating that silver forms an ohmic contact with AZO NRs. Given the nanorods were connected in parallel, the total resistances $\left(R_{\text {total }}\right)$ for the NRs having different lengths can be defined by $R_{\text {total }}=\frac{R_{N R}}{n}+R_{C}=\frac{\rho L}{A n}+R_{C}$, where $R_{N R}, n, R_{C}, \rho$, and $A$ denote the resistance of the single NR, the number of NRs available in the contact area, the contact resistance, resistivity of a single NR, and cross-section area of a single NR, respectively. Fig. $\underline{5 \text { shows temperature dependent resistance values estimated from } I-V \text { characteristics of the }}$ NRs. Assuming $\rho, n$ and $R_{C}$ constant for each length of nanorods samples, only $A$ and $L$ are left as variables. However, the diameter of the nanorods stays pretty much constant after nanorods becomes longer than about $200 \mathrm{~nm}$. Therefore, choosing the length of the NRs in the range from 300 to $545 \mathrm{~nm}$ can also allow us to assume that $A$ stays constant for the NRs we studied. Using this simple method, resistivity of a single NR can be determined out of the length dependent measurements. Observed resistivity value of about $12 \Omega \mathrm{cm}$ at room temperature is in good agreement with the previously reported resistivity of about $38 \Omega \mathrm{cm}$ for AZO thin films [10] and also they are comparable with the previously published data that are about $5 \Omega$-cm for $\mathrm{ZnO}$ NRs [11].

Figure 6 shows the temperature dependence of electrical resistivity of the NRs at different temperature points changing from 25 to $135{ }^{\circ} \mathrm{C}$. The resistivity follows well the 
Arrhenius law described as $\rho=A \exp \left(\frac{E_{a}}{k_{B} T}\right)$, where $A$ is a pre-exponential factor, $k_{B}$ is the Boltzmann constant and $E_{a}$ is the activation energy. As the temperature increases, resistivity slightly increases at first and decreases on increasing the temperature, which indicates semiconductor behavior of the NRs. By the best fitting of the data (solid line), we obtained the value of $E_{a}=0.14 \mathrm{eV}$ from the linear part of the measurements. This linear behavior of $\ln$ $\rho$ versus $1000 / T$ indicates that a thermally activated transport mechanism dominantly operates in that temperature regime. Although no $E_{a}$ was reported for AZO NRs in the literature as being acquired by other methods, which could be used for comparison, the obtained $E_{a}$ is reasonable. Note that there is a good agreement between our estimated $E_{a}$ for AZO NRs and the $E_{a}$ values reported for AZO thin films, which spans a wide range of values changing from $0.058 \mathrm{eV}$ up to about $0.32 \mathrm{eV}[10,12]$. It is also close to the value of $0.12 \mathrm{eV}$ reported for $\mathrm{ZnO} \mathrm{NRs}$ grown by metalorganic chemical vapor deposition [13]. It is well known that parameters such as carrier concentration, mobility, and defect energy levels can all affect $E_{a}$ [14]. Therefore, $E_{a}$ for AZO thin films and nanostructures can be quite different depending on the growth method and detailed processing conditions used. Overall, electrical properties of our GLAD AZO NRs agree well with previously reported values.

\section{Conclusion}

XRD, SEM, UV-vis-NIR spectroscopy, and I-V measurements are carried out on AZO NRs prepared by the GLAD method. The temperature-dependent I-V measurements revealed that resistivity of the GLAD AZO NRs is about $12 \Omega-\mathrm{cm}$. We also estimated an activation energy of $0.14 \mathrm{eV}$ from these measurements. Electrical conduction in the AZO NRs can be well expressed by a model that takes into account thermally activated transport mechanism. Our results indicate that simple NR growth and metal capping process presented in this work can be used to characterize electrical properties of a wide variety of nanostructures, which is 
otherwise difficult to achieve using conventional nanofabrication methods such as optical- or nano-lithography.

\section{Acknowledgements}

One of the authors (A. Y.) acknowledges support from the Turkish Academy of Sciences (2219). This work was supported by NASA (grant no: NNX09AW22A) and NSF (grant numbers: EPS-1003970 and 1159830). Authors also would like to thank Center for Innovative Technologies at UALR for the help of taking SEM images and measuring UV-visNIR spectra.

\section{References}

[1] Z. L. Wang, Adv. Funct. Mater., 18 (2008) 3553.

[2] A. B. Djurisic, A. M. C. Ng, X. Y. Chen, Progress in Quantum Electronics, 34 (2010) 191.

[3] H. Kind, H. Yan, B. Messer, M. Law, P. Yang, Adv. Mater. 14 (2002) 158.

[4] K. Takanezawa, K. Hirota , Q.-S. Wei, K. Tajima, K. Hashimoto, J. Phys. Chem. C, $111(2007) 7218$.

[5] J. Zhang, Y. Yang, B. Xu, F. Jiang, J. Li, J. Cryst. Growth 280 (2005) 509.

[6] P. Li, D. Wang, Z. Wei, Q. Peng, Y. Li, Chemistry - A European Journal 19 (2013) 3735 .

[7] H. Cansizoglu, M. F. Cansizoglu, F. Watanabe, T. Karabacak, ACS Appl. Mater. Interfaces. 6 (2014) 8673.

[8] J. W. Chiou et al., Appl. Phys. Lett., 85 (2004) 3220.

[9] I. Muniyandi, G. K. Mani, P. Shankar, J. B. B. Rayappann, Ceramics International 40 (2014) 7993.

[10] M. M. Rahman et al., Mater. Sci. Technol., 28 (2012) 329. 
[11] M. N. Jung et al., Appl. Phys. Lett., 94 (2009) 041906.

[12] S. Tewari, A. Bhatatacharjee, Pramana, 76 (2011) 153.

[13] J. Y. Park, H. Oh, J.-J. Kim, S. S. Kim,J. Cryst. Growth 287 (2006) 145.

[14] S. Majumdar, P. Banerji, J. Appl. Phys. 107 (2010) 063702. 


\section{Figures Captions:}

Fig. 1. XRD profiles of glancing angle deposited (GLAD) nanorods (NRs). The inset shows an enlarged region of XRD patterns.

Fig. 2. Top-view (bottom row) and cross-sectional (top row) SEM images of the GLAD NRs of (a) and (b) $300 \mathrm{~nm}$, (c) and (d) $385 \mathrm{~nm}$, (e) and (f) $470 \mathrm{~nm}$, (g) and (h) $545 \mathrm{~nm}$, respectively. Scale bars are $100 \mathrm{~nm}$.

Fig. 3. The plot $(\alpha h v)^{2}$ versus photon energy (hv) for the NRs deposited having different lengths.

Fig. 4. $I-V$ characteristic of the $\mathrm{Ag} / \mathrm{AZO} / \mathrm{Si} / \mathrm{Ag}$ structure. The inset displays the corresponding schematic current set-up. A novel capping process was used to introduce the top blanket Ag contact on the tips of NRs.

Fig. 5. Temperature-dependent resistance values estimated from $I-V$ characteristics of the $\underline{\text { NRs. }}$

Fig. 6. Plot of $\ln \rho$ against $1000 / \mathrm{T}$ for the NRs. 
Figures:

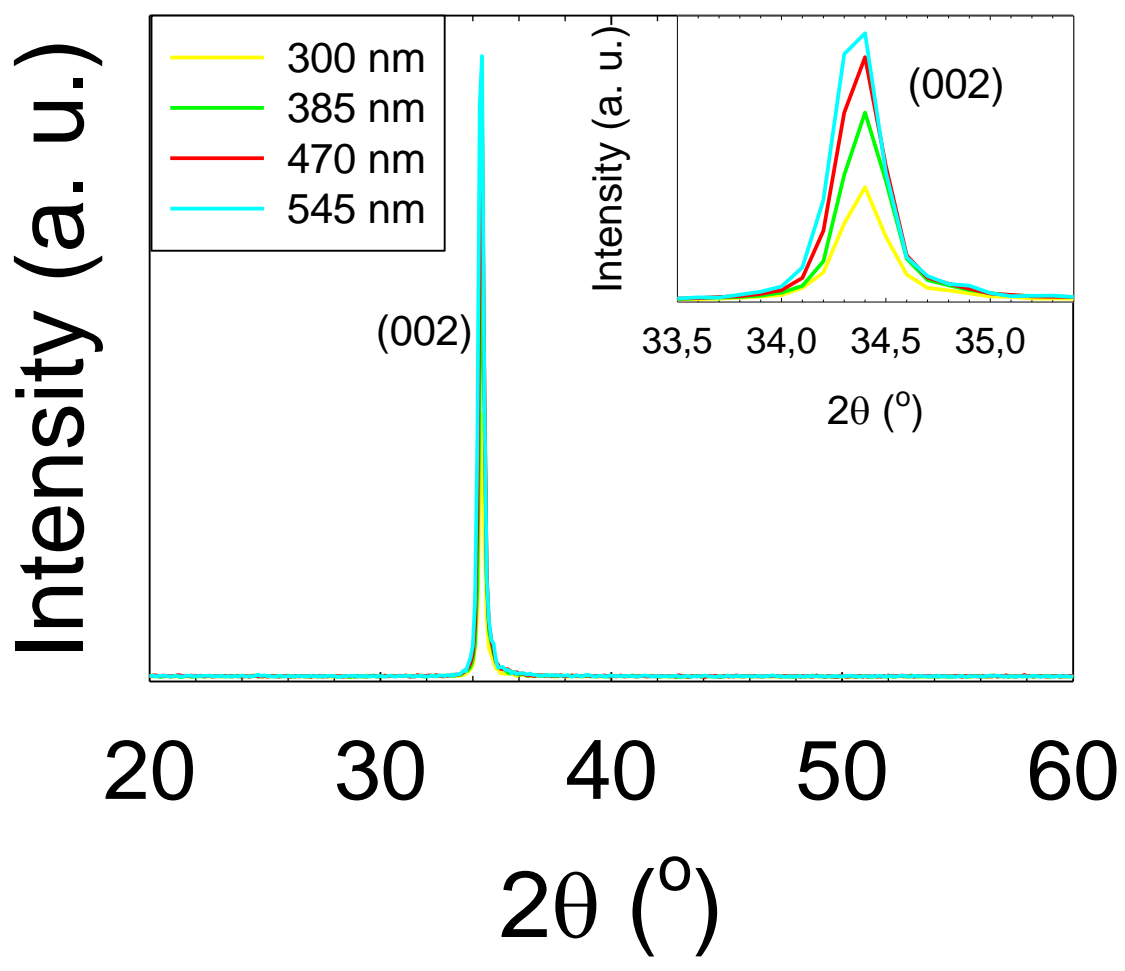

Fig. 1 


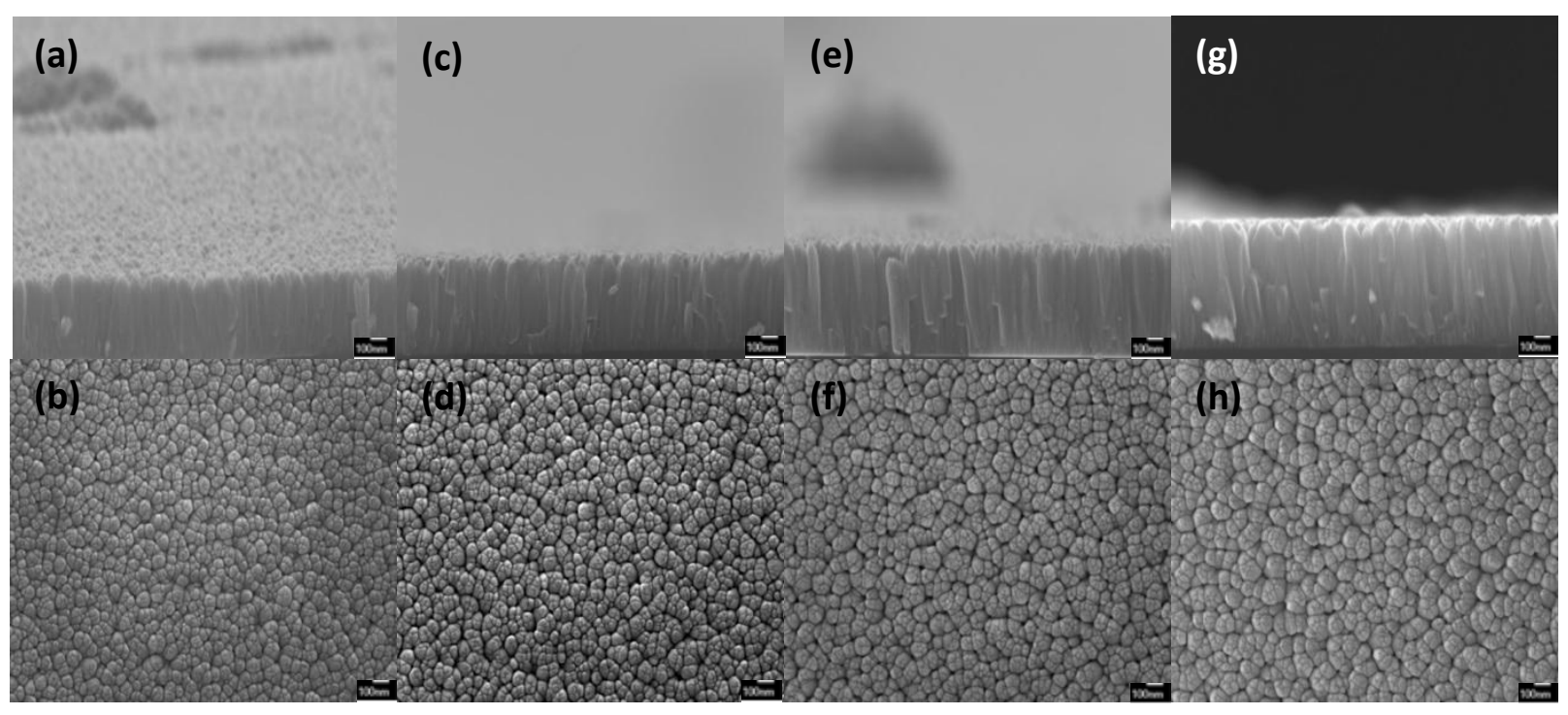

Fig. 2 


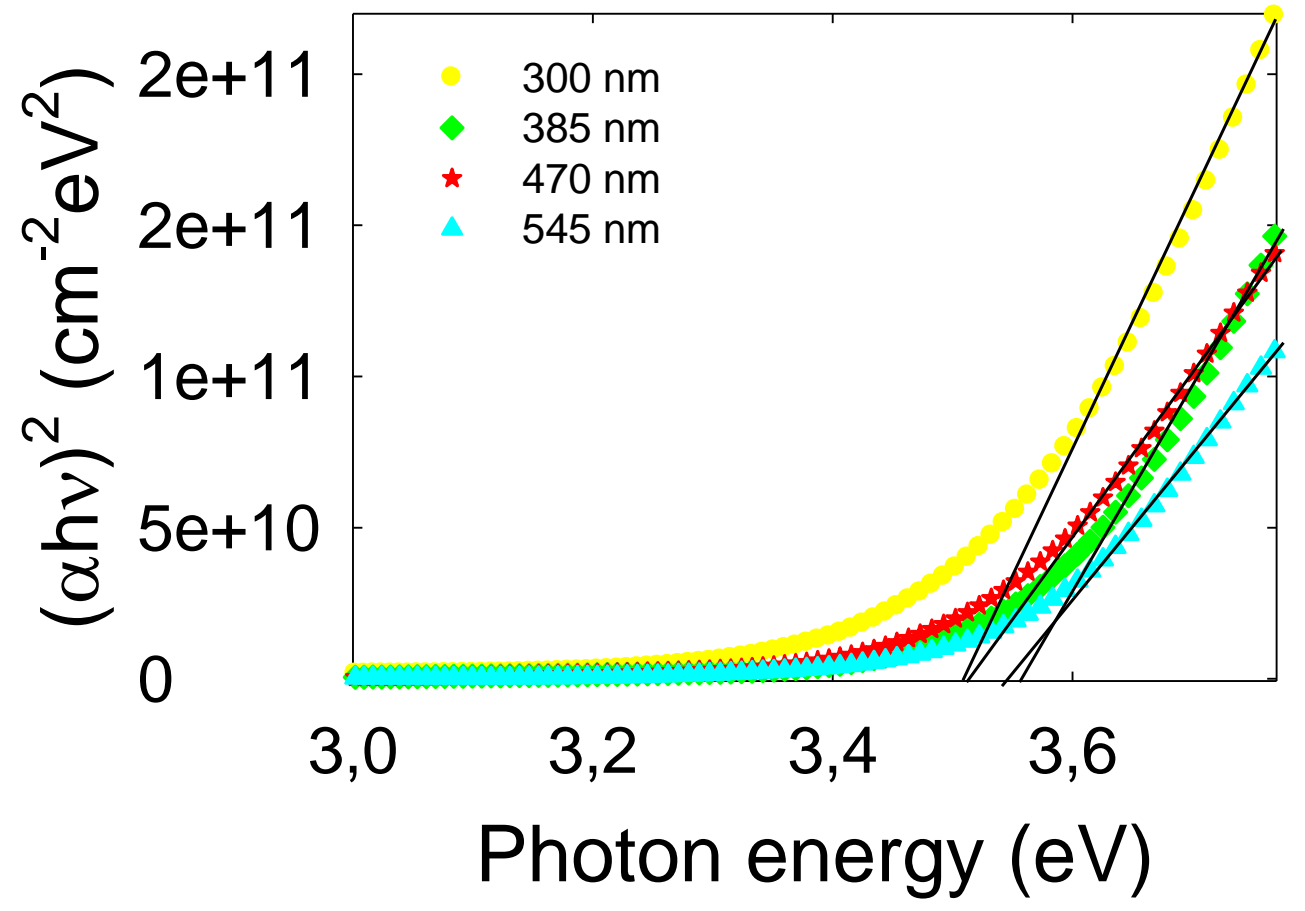

Fig. 3 


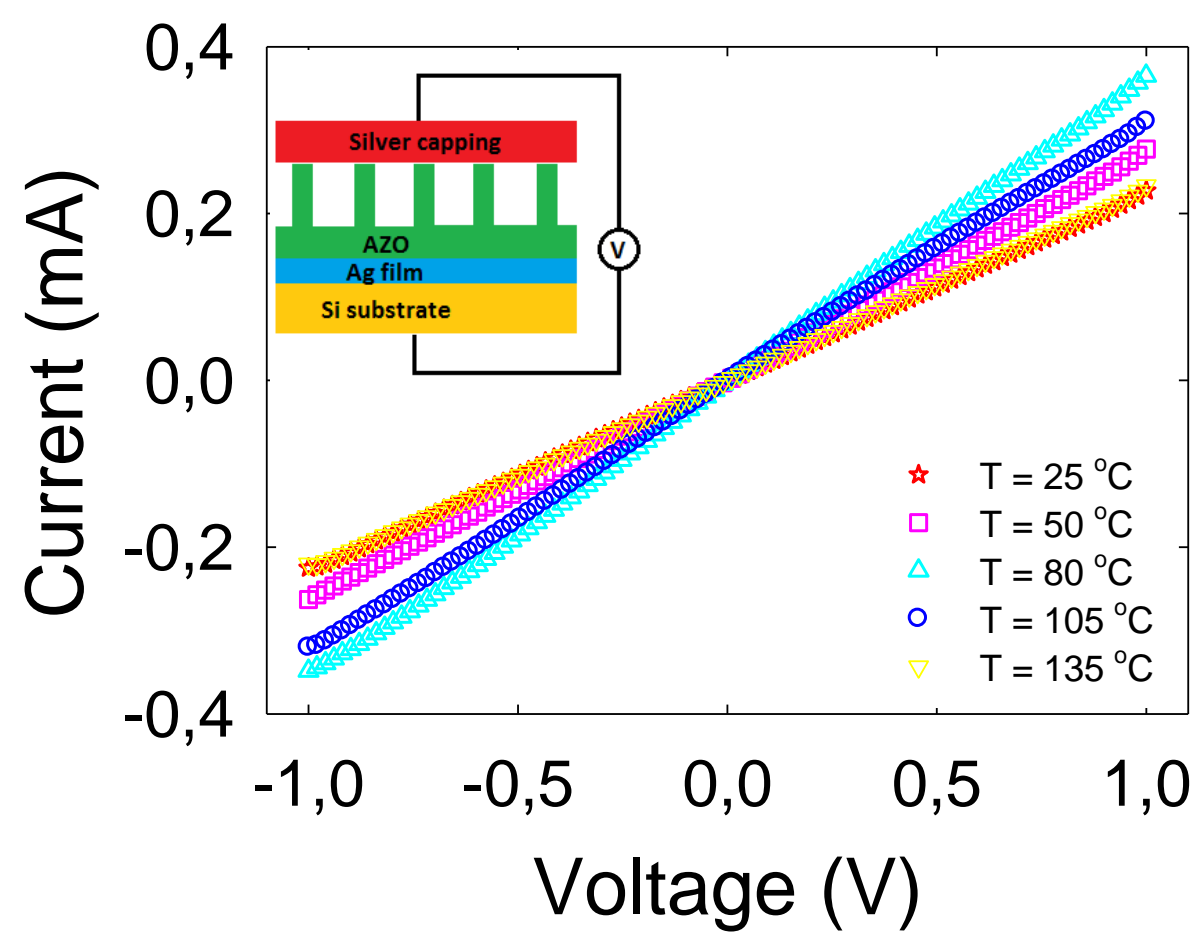

Fig. 4 


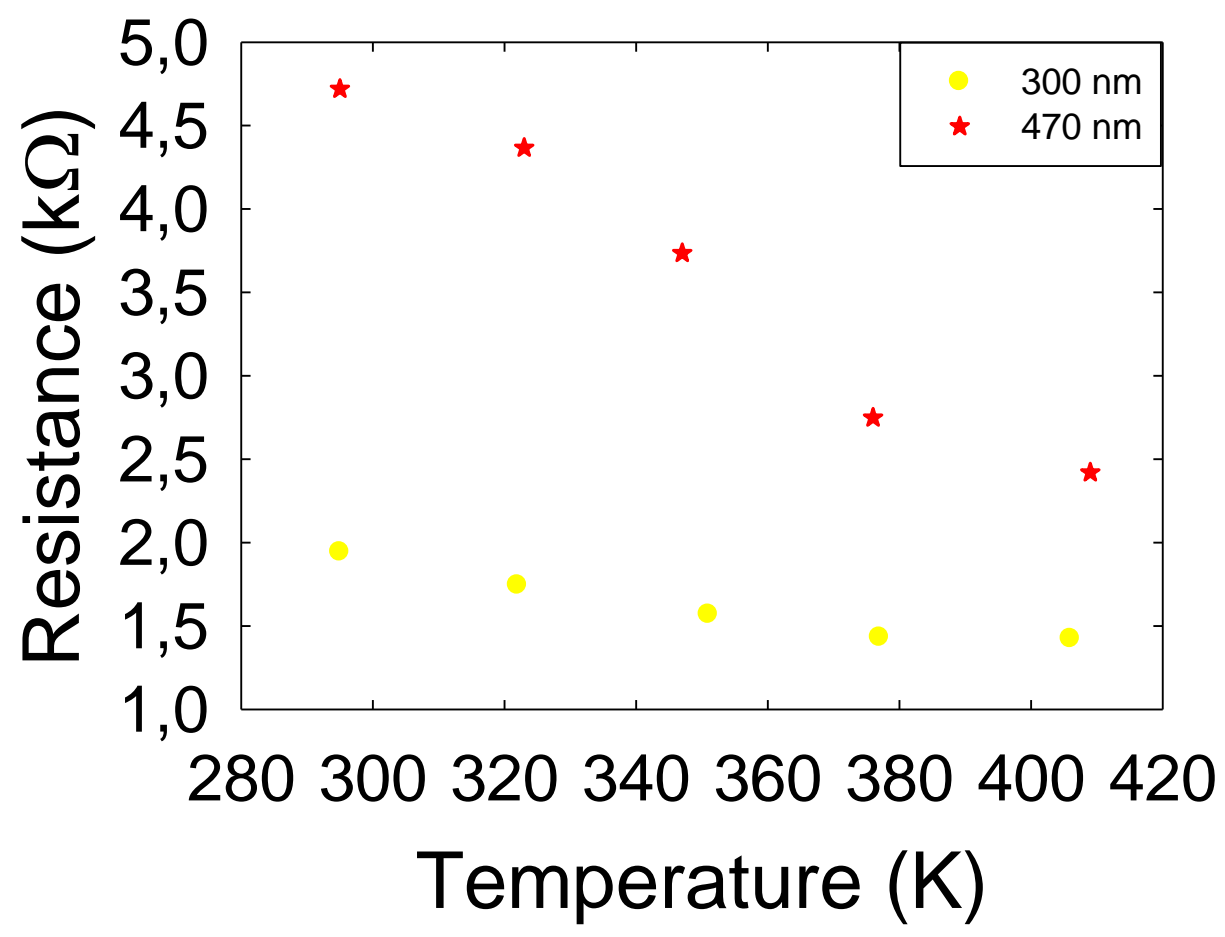

Fig. 5 


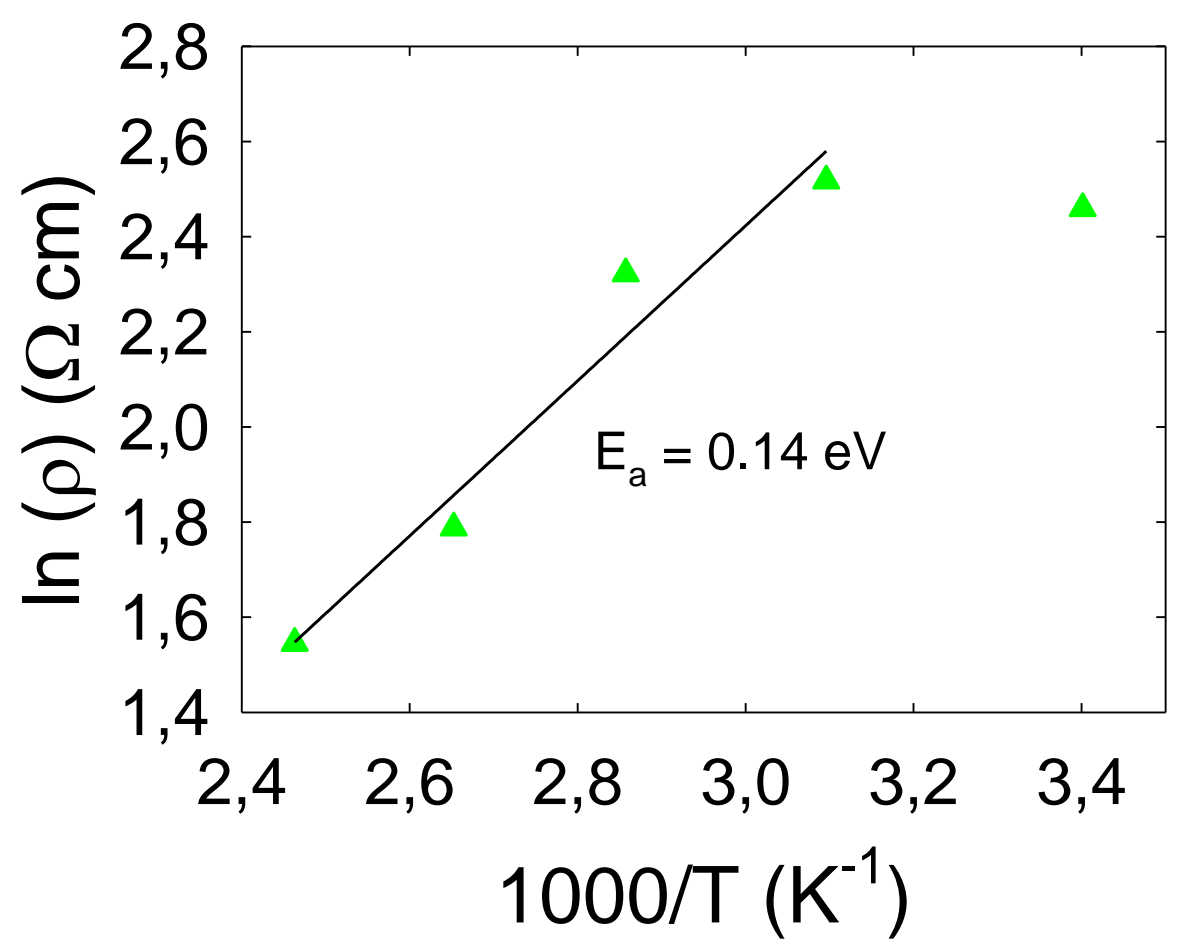

Fig. 6 